\title{
Imunidade: a importância de uma alimentação adequada em tempos de pandemia
}

\author{
Immunity: the importance of adequate nutrition in times of pandemic \\ Inmunidad: la importancia de una nutrición adecuada en tiempos de pandemia
}

Francielle Ribeiro Cavalcante ORCID: https://orcid.org/0000-0003-3419-6091

Faculdade Integrada Carajás, Brasil

E-mail: francielle16ccd@gmail.com

Naldina Luiza Líber

ORCID: https://orcid.org/0000-0003-3164-3722

Faculdade Integrada Carajás, Brasil

E-mail: naldina.luiza@gmail.com

Flávia Nunes Costa

ORCID: https://orcid.org/0000-0002-3486-9551

Faculdade Integrada Carajás, Brasil

E-mail: flaviaengal@gmail.com

\begin{abstract}
Resumo
O ato de alimentar-se é uma prática necessária para nossa existência. Sendo a alimentação saudável uma importante ferramenta para saúde e qualidade de vida da pessoa humana. Assim, o objetivo do presente estudo foi expor a importância da alimentação adequada na resposta imunológica em tempos de pandemia. Para tal, uma revisão bibliográfica de caráter descritivo com abordagem qualitativa foi realizada, onde selecionou-se artigos que abordavam a temática em um recorte temporal entre 2012 a 2021. O cenário pandêmico gerado pela COVID-19 reforçou a necessidade de políticas públicas que minimizem a insegurança alimentar e nutricional de famílias marginalizadas. Uma vez que, mesmo havendo legislação no Brasil que garanta o direito à alimentação saudável, em decorrência da crise econômica decorrente das medidas de isolamento e desemprego, tem-se observado impacto direto sobre o agravamento da situação de pobreza. É sabido que os alimentos nos fornecem nutrientes imprescindíveis para o fortalecimento do sistema imunológico, dentre esses estudos citam: a Vitamina A, Vitamina C, Vitamina D, Vitamina E, o Zinco, o Selênio, o Ferro e o Magnésio. Portanto, em tempos de pandemia alimentar-se de forma saudável é imprescindível. Nesse sentido, a escolha dos alimentos ingeridos deve priorizar os nutrientes presentes no alimento.
\end{abstract}

Palavras-chave: Alimentação; COVID-19; Sistema imunológico.

\begin{abstract}
The act of eating is a necessary practice for our existence. Healthy eating is an important tool for human health and quality of life. Thus, the aim of the present study was to expose the importance of adequate nutrition in the immune response in times of pandemic. To this end, a descriptive literature review with a qualitative approach was carried out, which selected articles that addressed the theme in a time frame between 2012 and 2021 . The pandemic scenario generated by COVID-19 reinforced the need for public policies that minimize the food and nutritional insecurity of marginalized families. Since, even with legislation in Brazil that guarantees the right to healthy food, as a result of the economic crisis resulting from the isolation and unemployment measures, there has been a direct impact on the worsening of the poverty situation. It is known that foods provide us with essential nutrients for strengthening the immune system, among these studies they mention: Vitamin A, Vitamin C, Vitamin D, Vitamin E, Zinc, Selenium, Iron and Magnesium. Therefore, in times of pandemic eating healthy is essential. In this sense, the choice of ingested foods should prioritize the nutrients present in the food.
\end{abstract}

Keywords: Food; COVID-19; Immune system.

\section{Resumen}

El acto de comer es una práctica necesaria de nuestra existencia. La alimentación saludable es una herramienta importante para la salud y la calidad de vida. Así, el objetivo de este estudio fue exponer la importancia de una nutrición adecuada en la respuesta inmune durante una pandemia. Para ello, se realizó una revisión descriptiva de la literatura con un enfoque cualitativo, en la que se seleccionaron artículos que abordaron el tema en el período de 2012 a 2021. El escenario pandémico provocado por el COVID-19 reforzó la necesidad de políticas públicas para reducir la alimentación. consumo. y la inseguridad alimentaria de los hogares marginados. Porque incluso en la legislación brasileña que garantiza el derecho a la alimentación saludable, la crisis económica derivada de las medidas de aislamiento y desempleo tiene un impacto directo en el agravamiento de la pobreza. Se sabe que los alimentos nos 
aportan nutrientes esenciales para fortalecer el sistema inmunológico, entre estos estudios se citan: vitamina A, vitamina $\mathrm{C}$, vitamina $\mathrm{D}$, vitamina $\mathrm{E}$, zinc, selenio, hierro y magnesio. Por lo tanto, durante una pandemia, una dieta saludable es esencial. En este contexto, los nutrientes presentes en los alimentos deben ser una prioridad a la hora de elegir los alimentos consumidos.

Palabras clave: Alimentación; COVID-19; Sistema inmunológico.

\section{Introdução}

A necessidade de alimentar-se faz parte da realidade humana desde sua existência. Consiste em uma prática fundamental para subsistência do homem. No entanto, o ato de comer consiste não somente para manutenção da vida, mas também se revela enquanto prática cultural (Cascudo, 2012).

Cascudo (2012) conceitua alimentação como necessidade básica e direito humano, mas não se limita somente a isso, esse conceito envolve vários outros fatores como escolhas, situação econômica, crenças e principalmente a cultura.

É sabido que os alimentos possuem um papel importantíssimo na manutenção da saúde humana, são as principais fontes de energia, minerais e vitaminas. Daí a necessidade de se ter uma alimentação saudável. Nesse sentido, uma alimentação equilibrada precisa conter porções de todos os grupos de alimentos como: proteínas, lipídeos, carboidratos, fibras, vitaminas e minerais. Isso significa que nenhum alimento sozinho fornecerá tudo que requer o organismo (Guimarães \& Oliveira, 2014).

É evidente que assim como a sociedade sofreu inúmeras transformações o hábito alimentar também acompanhou essas mudanças. Nos dias atuais, a alimentação tornou-se foco de vários estudos, isso pelo crescimento de alimentos industrializados que pouco contribuem para uma alimentação saudável. Esse novo modelo de comer da sociedade moderna, que vive na agitação e correria, tem comprometido a saúde dos indivíduos, tornando-os mais suscetíveis a doenças (Martinelli et al., 2020).

A pandemia da COVID-19, que se iniciou no Brasil em fevereiro de 2020, revelou a grande necessidade de uma alimentação rica em nutrientes, o que não é a prática de muitos brasileiros. Sendo declarada uma situação pandêmica pela Organização Mundial de Saúde (OMS) por se propagar em vários continentes de forma rápida e ao mesmo tempo. Mesmo havendo uma elevada capacidade de infecção, os casos mais graves apresentam-se em pessoas com doenças crônicas como diabetes, doenças respiratórias e cardiovasculares e pacientes acima de 70 anos. Doenças essas, em muitas situações consequentes de uma alimentação inadequada e desregrada, nesse aspecto, é importante salientar a relação estreita entre o ato alimentício e a saúde (Dultra et al., 2020).

Nesse sentido, mostrou-se necessário uma análise sobre a segurança alimentar e o direito à alimentação saudável, que preconiza as leis brasileiras diante da realidade socioeconômica de muitos indivíduos. Uma vez que, apresentar alimentos e seu valor nutricional, atendendo as diversas realidades, é imprescindível para contenção de doenças graves (Dultra et al., 2020).

Mesmo havendo poucos estudos sobre a COVID-19 ficou evidente que pacientes que possuem uma alimentação saudável possuíam uma baixa no índice de infecção e também uma boa resposta ao tratamento em detrimento do fortalecimento do sistema imunológico. Portanto, a alimentação tornou-se uma importante estratégia na prevenção do coronavírus, servindo como barreira imunológica (Lima Júnior, 2020).

Tendo em vista a importância de nutrientes como as vitaminas e minerais para o fortalecimento do sistema imunológico, é proposto nesse trabalho expor por meio de uma revisão integrativa da literatura a importância da alimentação adequada na resposta imunológica em tempos de pandemia.

O estudo é extrema relevância para todos indivíduos, sejam profissionais ou pessoas civis, isso porque a comensalidade é uma prática humana. Ademais, uma alimentação rica em nutrientes é impreenchível para manutenção da saúde e qualidade de vida. É inegável a contribuição dos alimentos para o corpo humano funcionar de forma harmonia, destacando que os nutrientes podem auxiliar no fortalecimento do sistema imune evitando, assim, doenças. 


\section{Metodologia}

O estudo consiste em uma revisão integrativa de literatura, de caráter descritiva e com abordagem qualitativa. Esse método de estudo visa a seleção de pesquisas científicas pertinentes a temática com o intuito de sistematiza a ideias de autores, ofertando acesso rápido e sintetizado aos resultados científicos de maior acuidade para a área estudada (Marconi \& Lakatos, 2018). Estabelecida por meio de artigos publicados que abordam a importância da alimentação para fortalecimento da imunidade em tempos de pandemia

A pesquisa foi realizada por meio documentos científicos publicados nas bases de dados Biblioteca Virtual em Saúde (BVS), Literatura Latino-Americana e do Caribe em Ciências da Saúde (LILACS) e Scientific Eletronic Library Online (SciELO). Realizou-se, também, consulta a sites da Organização Mundial da Saúde (OMS), Portarias e Leis do Ministério da Saúde e livros.

Após levantamento inicial, uma primeira seleção a partir dos títulos e resumos foi realizada e, após, realizou-se a leitura na íntegra, na qual, foram selecionados para elaboração do estudo 22 artigos, em um recorte temporal de 2012 a 2021.

Foram utilizados os critérios para inclusão os artigos sendo: publicados nos últimos 9 anos, preferencialmente em português e inglês que abordam a alimentação enquanto instrumento para atender os padrões de imunidade em tempos de pandemia e estão disponibilizados eletronicamente na íntegra. Os excluídos foram os artigos publicados fora do limite temporal 2012 a 2021, que não estejam disponíveis eletronicamente na íntegra e que não apresentaram relação com os objetivos da pesquisa.

Os descritores utilizados para a consulta às bases de dados: alimentação básica; segurança alimentar; COVID-19; vitaminas e minerais.

\section{Resultados e discussão}

\subsection{O homem e a alimentação}

A alimentação está presente na vida do homem desde sua existência, trata-se de uma necessidade básica. Nesse sentido, o hábito alimentar mudou conforme as transformações da sociedade, na atualidade o conceito de alimentar-se não se restringe apenas ao ato de comer, mas possui sentido amplo, envolvendo seleção, escolhas, disponibilidade, rituais entre outros aspectos sociais (Lima, Neto \& Farias, 2015).

A terminologia "alimento" surgiu por volta do ano 1120, mas o conceito material do termo se deu a partir do século XVI, quando houve a restrição da expressão "carne" na qual definia todo alimento comestível, limitando-se apenas como um tipo de alimento de origem animal (Lima, Neto \& Farias, 2015).

A história da alimentação abrange elementos como: tipo de alimentos, sua produção, distribuição, manuseio, preparo e consumo. A compreensão histórica de como um povo se alimenta é tão importante quanto o que se come, trata-se de uma representação cultural.

A cultura alimentar é uma das esferas presente na sociedade sendo fator de distinção entre os povos. A utilização e consumo de alimentos vegetais esteve bem presente nas primeiras civilizações, sendo pouco consumido carne e outros produtos derivados de animais. O que mudou consideravelmente com o decorrer e evolução da sociedade.

Conforme elucida o estudo de Lima, Neto e Farias (2015) a alimentação está bem além de uma atitude biológica, mas consiste em um comportamento reflexo de costumes de um povo. Os fatores biológicos remetem a sobrevivência da espécie humana, sendo condição absoluta para vida, a ingestão de alimentos é fundamental para manter o corpo em funcionamento. Já a representação cultural diz respeito aos hábitos de consumo que não é universal, diferencia-se conforme a cultura da sociedade. 
Os hábitos alimentares são adquiridos ainda na infância conforme a cultura dos pais os filhos passam a construir seu paladar alimentício. Nesse contexto, é importante ressaltar que não há uniformização quanto à escolha de alimentos, por isso que estudiosos elucidam que o ato de alimentar está diretamente relacionado com os fatores culturais.

O estudo de Cascudo (2012) afirma que a alimentação mudou de função conforme as transformações vividas pelo homem, o ato de comer passou a ter significado relacionado com outros fatores como rituais, religiosidade, festejos, entre outros.

Em conformidade Rodrigues (2012) afirma que: "[...] a comensalidade, o ato de comer juntos, é uma forma de começar uma relação ou de mantê-la. Ao mesmo tempo em que a refeição satisfaz uma necessidade humana essencial, ela é fator fundamental no desenvolvimento da identidade cultural de uma sociedade" (Rodrigues, 2012, p. 86).

Em suma, o ato de comer além de saciar o organismo o que é vital para o homem revela suas características culturais. Sendo assim, alimenta-se não se limita a atender as necessidades fisiológicas, mas reafirma e caracteriza uma sociedade distinguindo-a das demais.

Segundo Rodrigues (2012) a comensalidade está relacionada com o compartilhamento ou ação solidária entre os indivíduos. Para o homem a prática de alimentar-se não é individual e sim uma atividade social, isso porque a comida é compartilhada entre todos os entes familiares.

A alimentação nesse sentido possui vários papeis, o ato de banquetear explicita relações entre os indivíduos nas primeiras civilizações, na qual o banquete servia para integração entre os indivíduos. Sendo assim, o ato de comensalidade consistia no envolvimento dos participantes numa perspectiva solidária entre as famílias ou a comunidade. "O compartilhamento dos alimentos através da comensalidade também propicia a transmissão de valores entre seus participantes. Nestes momentos se expressam identidades e podem se operar mudanças sociais, pelo mecanismo antropológico conhecido do dom e da contrapartida" (Rodrigues, 2012, p. 90).

A solidariedade no ato alimentar diferencia o home dos demais animais, isso porque o animal alimenta-se exclusivamente por um instinto de sobrevivência, já o homem se "desarma" nesse momento e partilha com os demais do momento da refeição. A comensalidade humana é um momento de significados, sentidos, sentimentos e partilhas, não consiste apenas no ato de comer para saciar a fome (Cascudo, 2012).

Importante salientar também sobre os tipos de alimentos para serem consumidos na qual passam por uma seleção e eleição para serem preparados conforme os costumes culinários, tudo isso envolve a matéria cultural. No entanto, o fato da alimentação está relacionado com o modo de vida de um povo, com todos seus significados e ritos, não se pode dissociar sua importância ao processo vital (Silva, Prado, Seixas, 2017).

Comungando desse pensamento Vaz e Bennemann (2014, p. 2) afirmam que o "comportamento alimentar trata-se de algo muito complexo, pois comer é um ato social que vai para além das necessidades básicas de alimentação, indispensável ao desenvolvimento dos valores vitais, comum a todo ser humano". No momento de comer os indivíduos não buscam somente atender as necessidades fisiológicas, mas há uma relação de satisfação e prazer. Trata-se de relação mútua e não algo individual, a alimentação é uma ferramenta de interação.

Outro fator importante que envolve a alimentação diz respeito às classes sociais, a distribuição da riqueza no contexto social, conforme as condições financeiras têm-se a escolha dos alimentos a serem consumidos. Mesmo havendo leis que asseguram o direito à alimentação saudável a todos indivíduos é inegável a influência da questão social no processo alimentar (Silva, Prado \& Seixas, 2017).

\subsection{Segurança alimentar e nutricional em tempos de pandemia}

Desde os primórdios a alimentação é um requisito essencial a vida, ou seja, para manutenção da espécie é uma necessidade imprescindível para sobrevivência. Entende-se que para o homem desenvolver todas atividades humanas faz-se 
necessário uma alimentação equilibrada e saudável. Sendo assim, a alimentação é um direito humano. No entanto, diante da escassez, da miséria e das desigualdades sociais esse direito é negado a muitos indivíduos (Nascimento, 2020).

O conceito inicial de segurança alimentar remete o pós fim da Segunda Guerra Mundial (1939-1945), onde com economias devastadas pela guerra, a fome alastrou-se rapidamente. Com o intuito de conter esse avanço na Europa a discussão sobre o conceito ganha destaque envolvendo três aspectos principais: quantidade, qualidade e regularidade no acesso à alimentação (Nascimento, 2020).

O aspecto da quantidade remete a necessidade destacada pela Revolução Verde que consiste no aumento da produção utilizando metodologias moderna no setor agrícola. Nesse aspecto, passou a ser permitido o uso de agrotóxicos, fertilizantes, implementos agrícolas e máquinas (Nascimento, 2020).

No ano de 1945 foi instituída a Fundação da Organização das Nações Unidas Para Agricultura e Alimentação (FAO) que objetivava encontrar soluções para questão da alimentação visto que crescia a fome em países subdesenvolvidos.

A I Conferência Mundial de alimentação aconteceu na cidade de Roma e atribuiu o conceito de Segurança Alimentar como a possibilidade de todas as pessoas sem distinção temporal ter "[...] acesso físico e económico a alimentos seguros, nutritivos e suficientes para satisfazer as suas necessidades dietéticas e preferências alimentares, a fim de levarem uma vida activa (Gomes, 2020, p. 7)".

Em conformidade Vasconcellos e Moura (2018, p. 2) afirma: que A segurança alimentar e nutricional consiste na realização do direito ao acesso a alimentos de qualidade, em quantidade suficiente, sem comprometer outras necessidades essenciais".

Sendo assim, no Brasil, esse conceito se concretizou com o propósito de dar acesso a todos os brasileiros à alimentação com segurança, contribuindo para criação de leis, projetos, programas e planos que assegurassem a implantação da Segurança Alimentar e Nutricional (SAN). No ano de 2006 foi sancionada a lei no 11.346/2006 denominada Lei Orgânica de Segurança Alimentar e Nutricional-LOSAN na qual estabelece o conceito de SAN traçando as bases de constituição e funcionamento do Sistema Nacional de Segurança Alimentar e Nutricional-SINSAN (Gomes, 2020).

A discussão sobre o conceito de SAN está relacionada diretamente com a insegurança alimentar, ou seja, há uma preocupação desde o pós-guerra quanto a quantidade e distribuição dos gêneros alimentícios e se esses atendem a todos indivíduos do planeta. No entanto, conforme elucida a história, essa insegurança é bem presente em países subdesenvolvidos e populações marginalizadas que convivem ou com a fome ou com a limitação alimentícia.

O Brasil é um país de grande extensão territorial, sendo a maior parte das terras agricultáveis, o que coloca em discussão sobre o aumento da fome visto que o país é um dos maiores produtores de alimentos. No entanto, o modelo de produção agrícola utilizado por muitos produtores constitui-se na monocultura, ocupando extensas faixas de terra cultivando um produto único para atender o mercado de exportação o que eleva o preço dos alimentos, dificultando o acesso a pessoas de classe baixa. Em suma, no Brasil há suficiência de alimentos, porém ausência de políticas públicas para distribuição de renda (Campos et al., 2019).

Sendo assim, o que preconiza o art. $3^{\circ}$ da Lei $n^{\circ} 11.346 / 2006$ não se efetiva, pois, a lei assegura que a Segurança Alimentar e Nutricional consiste em um direito de todo indivíduo, possibilitando o acesso a alimentos saudáveis e em quantidades suficientes (Brasil, 2006).

As políticas públicas são fundamentais para reduzir as desigualdades sociais e promover as famílias em situação de vulnerabilidade. A questão alimentícia no país não está limitada a quantidade de alimentos produzidos, mas principalmente como se dá o acesso a estes. Percebe-se que em situação de pandemia esse problema aumenta, isso porque, as complicações econômicas decorrentes do momento pandêmico, torna a alimentação ainda mais escassa para algumas comunidades.

No ano de 2018 a FAO realizou um relatório sobre a SAN no mundo e divulgou que no ano de 2017 aproximadamente 5,2 milhões de pessoas passaram fome no Brasil. Portanto, o maior desafio do Brasil é criar mecanismos sustentáveis e 
autossuficientes de produção alimentar, além de propiciar o direito à alimentação a todos os cidadãos (Bocchi et al., 2019).

Importante salientar que a SAN integra os direitos humanos sendo denominado como Direito Humano à AlimentaçãoDHA como exemplifica Gomes Júnior (2015) ao afirmar que esse direito é imprescindível, inegociável, visto que a sua negativa traz riscos a existência humana.

A alimentação implica vários fatores como a disponibilidade de renda das pessoas para seu acesso, nesse sentido, é primordial desenvolver políticas públicas e econômicas para garantir esse indivíduo a todo brasileiro. A ausência de renda pode além de limitar o acesso, também traz outro problema que é a baixa qualidade nutricional, porque como confere a LOSAN não basta garantir o direito ao alimento é primordial possibilitar uma alimentação saudável e equilibrada.

O corpo humano requer para manutenção biológica nutrientes e alimentares que são ingeridos a partir de grupo de alimentos na qual o organismo extrai todos valores nutricionais necessários para subsistência. Destaca-se que há uma estreita relação entre a alimentação e a saúde da pessoa, daí a necessidade de uma alimentação adequada e saudável.

No Brasil um dos problemas de saúde bem presente decorrente da falta de acesso a alimentação é a desnutrição, a ausência de ingestão de micronutrientes como o ferro, cálcio e vitamina A e D trazem consequências a saúde comprometendo a qualidade de vida da pessoa. Segundo Silva et al., (2020, p. 3422) a "[...] anemia é um dos problemas nutricionais que representam riscos mais significativos para a saúde, particularmente quando ocorre durante a gravidez".

A pandemia da COVID-19 não é responsável absoluta pela fome ou evidencia a insegurança alimentar, no entanto, a sua contribuição para o aumento das desmazelas econômicas é inegável. Nesse aspecto, o distanciamento entre o que preconiza a legislação brasileira e o Direito Humano a Alimentação Adequada, com a pandemia, tornou-se um grande desafio sua efetivação (Aalpino et al., 2020).

A alimentação no período de pandemia apresenta dois gargalos: a alimentação e a nutricional. A primeira diz respeito à disponibilidade dos alimentos que com a crise econômico elevou-se os valores; a segunda refere-se a escolha, ao preparo e consumo dos alimentos e sua relação direta com os aspectos financeiros do cidadão.

As medidas de isolamento social adotadas pelos governos recomendada pela OMS comprometeram a empregabilidade de boa parte dos brasileiros. A agricultura familiar passou a oferecer menos alimentos frescos e comprometeu a produção o fechamento do comercio elevando assim, os valores dos produtos. Com a elevação dos preços famílias marginalizadas passaram a comprar produtos de baixa qualidade e valor nutricional, ou até mesmo deixaram de se alimentar por ausência de renda (Silva et al., 2020).

Em conformidade Silva et al. (2020, p. 3422) afirma em seus estudos que "[...] para muitos grupos da agricultura familiar, aqueles que já viviam em situação de pobreza ou limitações nas condições socioeconômicas, a pandemia da COVID19 pode significar intensificação da vulnerabilidade social”. Sendo assim, a pandemia não afetou somente a questão de saúde em si, mas trouxe consigo outros problemas de natureza social que não se dissociam da crise sanitária.

Alpino et al. (2020) explica que diante dos cortes no orçamento destinado a agricultura familiar, tornou-se quase impossível romper com os desafios da COVID-19. Outrossim, é que em decorrências das medidas de isolamento social houve um grande impacto sobre a cadeia de trabalho principalmente para o universo feminino. Ressaltando que a pandemia e as medidas de isolamento atingem de forma diferentes os indivíduos, afetando diretamente, em determinadas classes sociais a questão da insegurança alimentar.

A alimentação saudável ficou comprometida diante dos desafios e das medidas restritivas, o que aumentou consideravelmente o consumo por alimentos industrializados, por sua facilidade e praticidade para o consumo. Nesse aspecto, na qual é fundamental consumir alimentos balanceados com alto poder nutriente para fortalecer o sistema imunológico, o que se percebeu foi exatamente o contrário (Alpino et al., 2020).

Nesse sentido, a implantação de programas e políticas públicas voltadas a SAN para famílias em situação de 
vulnerabilidade social é imprescindível para garantir o direito mínimo de alimentar-se, não somente em pandemia, mas como o exercício de um direito humano fundamental para subsistência da vida humana. No entanto, o que se evidencia é a ausência de governos comprometidos em garantir os direitos fundamentais expressos na Carta Magna.

\subsection{Alimentação saudável e sua contribuição para o fortalecimento imunológico}

É sabido que uma boa alimentação é fundamental para qualidade de vida, para isso faz-se necessário uma seleção criteriosa dos nutrientes. Nesse sentido, o hábito alimentar é carregado de identidade, pois revela: cultura, crenças, valores e normas (Biasebetti, Rodrigues \& Mazur, 2018).

O primeiro passo para se ter uma alimentação saudável parte da seleção dos alimentos. Ingerir alimentos naturais e com alto valor nutricional tem sido cada vez mais desafiador diante dos "fast foods" bem presente na sociedade dos dias atuais. A influência dos alimentos industrializados compromete a qualidade na alimentação e contribui para doenças (Biasebetti, Rodrigues \& Mazur, 2018).

Nesse sentido, é importante desmitificar que alimentos saudáveis não são saborosos e para isso é fundamental o conhecimento sobre o valor nutricional dos alimentos e como manuseá-los para o consumo. Igualmente, essa conscientização deve alcançar pessoas com poucos recursos demonstrando que é possível se ter uma boa alimentação fazendo a escolha adequada dos alimentos e tornando o cardápio variado, saboroso e principalmente nutritivo, melhorando assim, a imunidade (Lima Júnior, 2020).

O estudo de Lima Júnior (2020) conceitua imunologia como um sistema composto por células como linfócitos, fagócitos e leucócitos que realizam a defesa do corpo humano contra fungos, vírus, bactérias e outros tipos de invasores. O sistema imunológico é responsável pela manutenção da saúde do indivíduo, nesse sentido, a alimentação contribuirá para manter a qualidade de vida.

Destaca-se que muitos fatores contribuem para imunidade dentre estes o sono, a atividade física, fatores emocionais e a alimentação. De modo que a alimentação não se limita ao ato de saciar a fome, mas nutrir-se com alimentos saudáveis é crucial para uma vida saudável e equilibrada. Consumir alimentos variados e com boa qualidade nutricional em quantidade adequada são fatores primordiais para saúde do indivíduo. Nesse sentido, nutrientes ricos em Vitamina A, C, Ferro, Zinco e Selênio podem atuar positivamente no sistema imunológico (Lima Júnior, 2020).

Estudos evidenciam que a vitamina A contribui para manter a integridade estrutural das células presentes na pele, função respiratória e outros. Sendo assim, a manutenção das células é importante para imunidade, isso porque, a referida vitamina é um importantíssimo antioxidante. Essa vitamina encontra-se em alimentos de origem animal (retinol) e vegetais de cor alaranjadas e verde-escuros (Costa et al., 2020).

$\mathrm{O}$ ácido ascórbico, forma ativa da vitamina $\mathrm{C}$, atua como um importantíssimo antioxidante, que potencializa o número de anticorpos atuante na proliferação de células do sistema imunológico. A vitamina $\mathrm{C}$ encontra-se presente principalmente nas frutas cítricas. Já o Ferro é imprescindível para diferenciação e crescimento celular, sendo um componente fundamental para o funcionamento de células imunes, está presente em alimentos de origem animal (ferro heme e não heme) e de origem vegetal (ferro não heme) são fontes de ferro (Costa et al., 2020).

Em conformidade Costa et al. (2020, p. 1) afirma que "a vitamina C é comumente encontrada em alimentos cítricos, como laranja e tangerina, compostas por antioxidantes e outras substâncias nutricionais”. Nesse sentido, as propriedades dessa vitamina disposta nos alimentos contribuem para prevenção de doenças, inflamações e envelhecimento precoce (Costa et al., 2020, p. 1).

Segundo a Agência Nacional de Vigilância (2005) recomenda-se que os brasileiros adultos consumam diariamente cerca de 45mg de ácido ascórbico. No entanto, contrariando essa recomendação estudos como o de Nascimento et al. (2018) afirmam 
que a maior parte da população brasileira não consome alimentos suficientes contendo essa vitamina o que compromete a qualidade de vida e a saúde dos cidadãos (Nascimento et al., 2018).

O estudo de Nascimento et al. (2018) destaca que o processamento dos alimentos ricos em vitamina C reduz os antioxidantes presente nos mesmos, ou seja, há uma perca do valor nutricional. Nesse sentido, percebe-se o crescimento por produtos processados, isso pela sua praticidade e durabilidade, porém o consumidor deve estar atento a questão nutricional que nesse caso é precária.

No ano de 1971 o pesquisador Linus Pauling evidenciou que a vitamina C reduz a "morbidade integrada" dos resfriados, em relação a outros pacientes que possuíam carência dessa suplementação. Importante salientar que essa redução não foi percebível somente em pessoas que praticam constantemente atividades físicas. A vitamina $\mathrm{C}$ também reduz a duração e a gravidade dos sintomas de resfriados comum, sendo um forte anti-histamínico (Costa et al., 2020).

O Zinco assim como a vitamina $\mathrm{C}$ é um antioxidante e exerce um papel de diferenciação e renovação de células imunes. Encontra-se nos alimentos de origem animal, nas sementes e castanhas. "O zinco atua no controle das vias de sinalização celular da imunidade inata e adaptativa" (Martins \& Oliveira \& 2020, p. 17). Segundo os autores as principais fontes de zinco estão dispostas nas ostras, carne bonina e principalmente nas vísceras como fígado e rins, os frutos do mar, as oleaginosas, os cereais integrais, as leguminosas e o leite.

Para Martins e Oliveira (2020) o zinco contribui para atividades biológicas fundamentais para saúde da pessoa, atuando como regulador enzimático. Realiza, ainda, o transporte da vitamina A, sendo importantíssimo na síntese hepática. Trata-se de um mineral anti-inflamatório que integra o sistema de defesa antioxidante.

No sistema imunológico o zinco tem uma função especial que consiste na proliferação e maturação das células de defesa, portanto, a carência desse mineral pode acarretar suscetíveis infecções. Outrossim, é que a célula Killer (NK), que é necessária para fortalecer a imunidade contra infecções e tumores, necessitam do zinco para o reconhecimento de moléculas de histocompatibilidade da classe I (MHC) (Biasebetti, Rodrigues \& Mazur, 2018).

O selênio atua diretamente na regulação do sistema imunológico, constitui-se um oligoelemento imprescindível a saúde do indivíduo. É encontrado em diversos ambientes em quantidades variadas, os oleaginosos como Castanha do Pará, feijão, especialmente o feijão preto e vermelho, e também as farinhas como farinha integral de trigo e de fubá, nas carnes preferencialmente no filé bovino e no fígado de galinha. Os peixes como a sardinha e o atum possuem altas concentrações de selênio, além de outros alimentos como o ovo e o leite (Santiago \& Souza, 2020).

O estudo de Santiago e Souza (2020) evidenciam que a ausência de Selênio-(SE) no organismo torna o indivíduo mais propenso a contrair doenças virais, isso porque ele fortalece o sistema de defesa do organismo. Sendo assim, fica evidente que a baixa do nutriente pode influenciar na infecção por vírus. Assim sendo, "seus benefícios à saúde vão desde a ação antioxidante até a formação de hormônios da tireoide, hormônios sexuais, contribuição na fertilidade e gestação, entre outros. Em quantidades insuficientes, a baixa absorção de se pode tornar humanos e animais inférteis, e nas deficiências graves levar a anomalias e distúrbios de saúde (Santiago \& Souza, 2020, p. 12).

O selênio regula os hormônios que são vitais para saúde, nesse sentido, uma alimentação equilibrada quanti e qualitativamente pode ser o grande diferencial para evitar doenças. A alimentação tem um papel importantíssimo na condição de a saúde do homem, sendo fator preponderante no fortalecimento do sistema imunológico (Lima Júnior, 2020).

Conforme alude Rodrigues et al. (2021) a vitamina D possui micronutrientes essenciais para os processos imunobiológicos sendo fundamental para a saúde humana. De modo que a Vitamina D regula o ciclo celular o que é imprescindível para fortalecimento do sistema imune atuando como imunomodulador nas doenças. 
Para Silvino et al. (2020) a Vitamina D é essencial para o sistema imune, a mesma pode ser obtida na alimentação e por meio de fotorreação mediada pela luz solar. Os alimentos de fontes de origem vegetal e fontes animais, conta de seus efeitos positivos sobre a imunidade e no combate de infecções virais do trato respiratório superior.

Segundo Lima et al. (2020) a Vitamina E é um importante antioxidante importantíssimo para saúde humana, trata-se de um nutriente com alta capacidade de modulação do sistema imune. De modo que, pode ser encontrada nos óleos de soja, milho, gérmen de trigo e palma. Em suma, essa vitamina contribui para proteção dos ácidos graxos poli-insaturados da membrana plasmática, e no sistema imunológico atua na multiplicação de quantidade de linfócitos.

O magnésio contribui no processo de produção de proteínas servindo de transporte de energia mantendo o bom funcionamento celular. Esse micronutriente está envolvido na síntese de nucleótidos e ácidos nucleicos. Sendo assim, trata-se de um importantíssimo nutriente no papel de maturação, diferenciação e fortalecimento das células imunitárias (Ferraz, 2021).

Sendo assim, nutrir-se fazendo uma seleção adequada dos alimentos é um componente crucial para saúde humana. Verifica-se que a alimentação saudável e regrada torna-se uma importante estratégia para prevenção e recuperação após doenças. Portanto, o conhecimento sobre quais alimentos devemos inferir para aumentar a imunidade é imprescindível para tomada de decisão no processo de escolha alimentar (Ferraz, 2021).

O Quadro 1 seguinte exemplifica as vitaminas e sais minerais que contribuem para o aumento da resposta imune e em quais alimentos encontramos: 
Quadro 1: Vitaminas e minerais importantes para o sistema imunológico e os alimentos onde são encontrados.

\begin{tabular}{|c|c|c|}
\hline NUTRIENTE & CONTRIBUIÇÃ̃O & ALIMENTOS \\
\hline Vitamina A & $\begin{array}{c}\text { Mantém a integridade das células da pele, } \\
\text { do trato respiratório e outros tecidos, } \\
\text { sistemas ou órgãos. }\end{array}$ & $\begin{array}{l}\text {-Ovos } \\
\text {-Leite e queijos; } \\
\text {-Fígado; } \\
\text {-Legumes, verduras e frutas cor alaranjada: abóbora, } \\
\text { mamão, manga e cenoura; } \\
\text {-Legumes e verduras de cor verde-escuros: agrião, } \\
\text { couve, espinafre e rúcula. }\end{array}$ \\
\hline Vitamina $\mathbf{C}$ & $\begin{array}{l}\text { Importante antioxidante, aumenta o número } \\
\text { de anticorpos e atua na diferenciação e } \\
\text { proliferação de células do sistema imune. }\end{array}$ & -Frutas cítricas: laranja, limão, mexerica e acerola. \\
\hline Vitamina D & $\begin{array}{l}\text { Atua no funcionamento ideal de muitos } \\
\text { órgãos e tecidos, além, de possuir } \\
\text { importância para a saúde dos ossos. Tem } \\
\text { também efeitos significativos no cérebro, } \\
\text { próstata, mama, cólon, coração, células do } \\
\text { sistema imunitário, pâncreas e sistema } \\
\text { vascular. }\end{array}$ & $\begin{array}{l}\text {-Exposição aos raios solares; } \\
\text {-Óleos de fígado de peixes: atum, linguado, bacalhau, } \\
\text { salmão, cavala e sardinha; }\end{array}$ \\
\hline Vitamina $\mathbf{E}$ & $\begin{array}{c}\text { Atua como antioxidante e neutraliza os } \\
\text { radicais livres. }\end{array}$ & $\begin{array}{l}\text {-Gema de ovo; } \\
\text {-Vegetais verde-escuros; } \\
\text {-Sementes oleaginosas; } \\
\text {-Óleos vegetais; } \\
\text {-Germe de trigo; }\end{array}$ \\
\hline Ferro & $\begin{array}{l}\text { Principal componente da hemoglobina } \\
\text { (proteína responsável pelo transporte de } \\
\text { oxigênio no sangue). }\end{array}$ & $\begin{array}{l}\text {-Alimentos de origem animal: carnes, peixes e ovos; } \\
\text {-Alimentos de origem vegetal: feijão, lentilha, } \\
\text { castanhas, gergelim e linhaça. }\end{array}$ \\
\hline Magnésio & $\begin{array}{l}\text { Participa na produção de proteínas, } \\
\text { transporte de energia, na bomba de potássio } \\
\text { e sódio, equilíbrio do cálcio e no bom } \\
\text { funcionamento das células. }\end{array}$ & $\begin{array}{l}\text {-Cereais integrais e nozes; } \\
\text {-Vegetais folhosos verdes: espinafre, alface, couve, } \\
\text { rúcula e repolho; } \\
\text {-Frutas e legumes (tubérculos). }\end{array}$ \\
\hline Selênio & $\begin{array}{c}\text { Forte antioxidante, melhora a imunidade do } \\
\text { corpo e acelera a cicatrização do } \\
\text { organismo. }\end{array}$ & $\begin{array}{l}\text {-Castanha do Pará; } \\
\text {-Trigo; } \\
\text {-Arroz; } \\
\text {-Gema de ovo e frango; } \\
\text {-Sementes de girassol; }\end{array}$ \\
\hline Zinco & $\begin{array}{l}\text { Auxilia o organismo na atuação contra } \\
\text { resfriados, gripes e outras doenças. A } \\
\text { deficiência de zinco acarreta uma maior } \\
\text { fragilidade às infecções virais. }\end{array}$ & $\begin{array}{l}\text {-Azeite; } \\
\text {-Salmão e atum; } \\
\text {-Chia e linhaça; } \\
\text {-Ostras e camarão; } \\
\text {-Gema de ovo; } \\
\text {-Leite integral; } \\
\text {-Amendoim e castanha de caju. }\end{array}$ \\
\hline
\end{tabular}

Fonte: Cavalcanti (2020, p. 9).

Diante do quadro fica evidente que não se trata apenas de comer, é preciso fazer uma boa escolha dos alimentos a serem consumidos, isso para garantir a qualidade de vida. Nesse sentido, a SAN deve atender os preceitos de seleção nutritiva e possibilitar a todos os indivíduos o acesso a alimentos saudáveis e que atendam às necessidades nutricionais do indivíduo.

Em suma, uma alimentação saudável requer uma dieta balanceada consumindo alimentos nutritivos e sem excessos. Entende-se que o sistema imunológico é o responsável por defender o corpo dos invasores como os vírus e para isso ele precisa estar forte e nutrido. Nesse aspecto, a alimentação tem um papel importantíssimo para saúde humana.

\subsubsection{A COVID-19 e as implicações quanto a imunologia}

O primeiro indicio do novo coronavírus (SARS-CoV-2) aconteceu na China em dezembro de 2019, na qual expandiuse para outros países se transformando na pandemia de COVID-19, o que nos trouxe uma crise sanitária global. Em 30 de janeiro de 2020 a OMS decretou Emergência de Saúde Pública de Importância Internacional e uma pandemia em 11 de março de 2020 (Aquino \& Lima, 2020). 
Apesar de não haver estudos decisivos sobre essa doença, considerando sua alta transmissibilidade várias medidas foram tomadas com o objetivo de contê-la e reduzir o índice de contaminação, e uma destas é o isolamento social. As medidas incluíram isolamento social, higienização de mãos, uso de máscaras e principalmente redução de pessoas em locais para não gerar aglomeração e consequentemente o contágio (Aquino \& Lima, 2020).

Tais medidas foram adotadas gradualmente atendendo as necessidades da população local, não sendo essas medidas iguais para todos os países. No Brasil, especificamente os Estados e Municípios, após decisão do Supremo Tribunal Federal, passaram a decidir sobre as medidas de isolamento social conforme a realidade dos mesmos (Pires, 2020).

No entanto, é importante frisar que para efetivação dessas medidas é preciso considerar os aspectos socioeconômicos, culturais, a administração política em saúde e ao apoio da sociedade, bem como todos recursos operacionais que são necessários para sua implementação (Martins \& Oliveira \& Sales, 2020).

Sendo assim, atentando-se a realidade do Brasil, é preciso destacar a desigualdade social e regionais na qual temos, são 66 milhões de pessoas que vivem abaixo da linha da pobreza, tendo somente $40 \%$ da população com emprego formal. Portanto, as medidas de isolamento social tiveram grande impacto no país (Reis \& Macagnan, 2020).

O desafio de fazer o isolamento social e a questão da desigualdade econômica é a trave para a efetivação dessa medida. Muitas famílias não possuem condições financeiras para manter-se em casa, daí a necessidade de políticas públicas que sanasse essa problemática (Pires, 2020).

Segundo o estudo de Pires (2020) na cidade de São Paulo, e especialmente na favela de Heliópolis 68\% das famílias tiveram perdas no rendimento mensal em detrimento das medidas de isolamento e destas, $20 \%$ não possuem renda nenhuma. Importante salientar, que dentre as pessoas que mais perderam renda está a mulher que é mais suscetível ao desemprego.

As medidas de isolamento eram a única garantia de controle da doença. Isso porque ainda não há tratamento eficaz normatizado para essa COVID-19. No entanto, já estão vacinando a população, o que reduziu drasticamente o número de casos graves e óbitos. Importante salientar que os estudos são unânimes quanto a alimentação saudável como estratégia para fortalecimento do sistema imunológico (Gois et al., 2020).

Em conformidade Gois et al. (2020, p. 91) afirma que "A alimentação deve ser variada e composta por alimentos de boa qualidade nutricional. A quantidade deve ser adequada, uma vez que tanto a sub quanto a hiper nutrição, são igualmente prejudiciais". Nesse sentido, é recomendável uma alimentação preferencialmente in natura evitando os alimentos processados, pois estes possuem baixíssima qualidade nutricional.

Com as medidas de isolamento cresceram significativamente a procura por alimentos ultraprocessados, os fast food, no entanto recomenda-se que devem ser evitados esses tipos de alimentos. Os alimentos in natura possuem um valor nutritivo maior destacando que trazem muitos benefícios a saúde. Contrariamente, uma alimentação desequilibrada quanti e qualitativamente contribui para proliferação de doenças, especialmente das Doenças Crônicas Não Transmissíveis-DCNTs. As DCNTs englobam um conjunto de enfermidades como: obesidade, câncer, diabetes, hipertensão arterial, entre outras que reduzem a qualidade e expectativa de vida (Santiago \& Souza, 2020).

Em relação a COVID-19 estudos evidenciaram que alguns nutrientes como Vitamina A, E, D, C, Ferro, Selênio e Zinco contribuem positivamente para o fortalecimento do sistema imunológico e é possível atingir as necessidades diárias desses nutrientes com a alimentação saudável (Santiago \& Souza, 2020).

Segundo Dias et al. (2020) esses nutrientes apoiam o desenvolvimento do sistema imune servindo como barreiras contra doenças. A alimentação saudável constitui-se um importante remédio natural que além de fortalecer o sistema imune traz qualidade de vida a pessoa. 
No entanto, é importante frisar que esses alimentos não prometem a cura da COVID-19, mas auxiliam no sistema imune no combate às doenças. Nesse sentido, a alimentação é um aliado indispensável para qualidade de vida. Nesse sentido, é inquestionável os benefícios de uma alimentação rica em nutrientes (Gois et al., 2020).

A vitamina $\mathrm{C}$ foi uma das mais procuradas na pandemia, isso porque, possui numerosos efeitos no sistema imunológico, por isso, pesquisadores evidenciaram sua atuação em pacientes com COVID-19. Segundo Dias et al. (2020) a ingestão regularmente de 1g/dia de Vitamina C é fundamental para redução dos sintomas de infecções respiratórias. Estudos evidenciaram que a vitamina $\mathrm{C}$ aumenta a resistência de culturas de órgãos traqueais em embriões de pintos a infecções por coronavírus aviário. Comprovou-se, ainda, sua eficácia como anti-histamínico fraco para aliviar sintomas gripais como espirros, coriza e seios nasais inchados (Dias et al., 2020).

Outro importantíssimo metal necessário para prevenção da COVID-19 citado em estudos é o zinco devido ao seu envolvimento em vários processos biológicos, sendo um dos responsáveis por modular a imunidade antiviral e antibacteriana. $\mathrm{O}$ zinco contribui significativamente para melhoria do sistema imunológico o que é fundamental para prevenir doenças (Martins, Oliveira \& Sales, 2020).

Sendo assim, Martins et al. (2020) reafirma a importância do zinco para saúde, isso porque, "o zinco tem um efeito importante em doenças virais por meio de mecanismos de modulação da entrada de vírions, internalização do vírus, duplicação do material genético, biossíntese de proteínas virais e liberação adicional de diversas partículas virais, incluindo os relacionados com as doenças do trato respiratório (Martins, Oliveira \& Sales, 2020, p. 7)”.

No que diz respeito a COVID-19 estudos evidenciaram que o zinco melhora os sintomas da doença, isso porque é um oligoelemento com potencial terapêutico em decorrência de sua contribuição para modulação do sistema imunológico, ou seja, sua atividade é direta contra o vírus (Martins, Oliveira \& Sales, 2020).

Estudos associaram a deficiência de selênio e a COVID-19 em pesquisa com um grupo de não sobreviventes ficando evidente e comum entre os casos a grave deficiência de selênio, sendo assim, quanto mais baixa os níveis desse mineral mais expostos a complicações da COVID-19 está o indivíduo. Evidenciou-se que a ausência de selênio está relacionada com evolução de doenças infecto virais (Santiago \& Souza, 2020).

O selênio é um mineral antioxidante com propriedades anti-inflamatórias sendo importantíssima para função imune. "Ainda, segundo a pesquisa, o selênio teria a propriedade de reduzir a ocorrência de trombose (Dias et al., 2020, p. 111)". Nesse aspecto, o selênio contribui para terapia anticoagulante reduzindo a formação de coágulos, um dos fatores de morte em pacientes com COVID-19.

Assim, fica evidente que uma alimentação adequada e saudável tem um papel fundamental na prevenção e combate de doenças. No entanto, é preciso destacar que ainda existem famílias que não tem condições financeiras para ter uma alimentação diversificada e rica em nutrientes. Portanto, a implementação de políticas públicas é imprescindível para garantir ao cidadão brasileiro seu direito previsto na Constituição Federal.

\section{Considerações Finais}

A pandemia da COVID-19 é uma ameaça real a vida humana, isso porque não existe tratamento específico que garanta sua cura. Apesar de haver vacina, as medidas de prevenção são imprescindíveis, isso porque a vacina tem se apresentado como instrumento para redução de casos graves da doença.

Com base no que foi exposto no trabalho, a alimentação adequada e saudável se mostrou uma ferramenta importante para garantir suporte nutricional e o fortalecimento do sistema imunológico, o que é essencial em tempos pandêmicos. Citandose a contribuição das vitaminas A, C, D, E e dos minerais Ferro, Magnésio, Selênio e Zinco. Além da exemplificação dos alimentos que os possuem. 
No que diz respeito o acesso aos alimentos, como assegura a legislação, faz-se necessário a ampliação das políticas públicas que promovam famílias de baixa renda. Visto o avanço da insegurança alimentar agravada pela pandemia.

Assim, a promoção e adoção de práticas alimentares saudáveis é indispensável para o sistema imunológico, bem como na prevenção e enfrentamento da COVID-19.

O estudo sobre alimentos que contribuem para o fortalecimento do sistema imunológico é de extrema importância para sociedade civil, acadêmica e de profissionais que atuam na área, principalmente Nutrição, nesse sentido, recomenda-se o aprofundamento de pesquisas sobre a temática, especialmente as experimentais que apresentam dados estatísticos.

\section{Referências}

Alpino, T. de M. A. Santos, C. R. B. Barros, D. C. \& Freitas, C. M. de. (2020). COVID-19 e (in)segurança alimentar e nutricional: ações do governo Federal brasileiro na pandemia frente aos desmontes orçamentários e institucionais. Cad. Saúde Pública, 36(8):e00161320.

Aquino, E. M. L. \& Lima, R. T. dos R. (2020) Medidas de distanciamento social no controle da pandemia de COVID-19: potenciais impactos e desafios no Brasil. Ciência Saúde Coletiva. 25(Supl.1):2423-2446

Biasebetti, M. do B. C. Rodrigues, I. S., \& Mazur, C. E. (2018) Relação do consumo de vitaminas e minerais com o sistema imunitário: uma breve revisão. Visão Acadêmica, 19(1).

Bocchi, C. P. Magalhães, E. de S. Rahal, L. Gentil, P. \& Gonçalves, R. de S. (2019). A década da nutrição, a política de segurança alimentar e nutricional e as compras públicas da agricultura familiar. Rev Panam Salud Publica. 43, e84. https://doi.org/10.26633/RPSP.2019.84

Brasil, Lei no 11.346 de 15 de Setembro de 2006. http://www.planalto.gov.br/ccivil_03/_ato2004-2006/2006/lei/111346.htm

Campos, M. M. Rocha, D. F., Falçoni, S. F. S., Pacheco, L. D., Aalmeida, T. D. de., Rangel, C. S., Pagoraro, M. A. da S., Abreu, I. S., Siqueira, M. H., \& Barros, B. L. de J. R. (2019). Políticas públicas de segurança alimentar e nutricional em âmbito local: discussões e resultados de um projeto de extensão em campos dos Goytacazes (RJ). https://dialnet.unirioja.es/servlet/articulo?codigo=7154452.

Cascudo, L. da C. (2012). A alimentação. Ed. Global Editora.

Cavalcanti, I. M. F. (2020). Alimentação, Imunidade e covid-19. Ed. RFB Editora.

Costa, E. S. Moura, I. de A. Santos, G. F. C. Prado, L. G. V. B. \& Pascoal, D. (2020). A relação da vitamina C com o fortalecimento do sistema imunológico. Universidade Católica do Salvador | Anais da 23 Semana de Mobilização Científica- SEMOC | 2020. http://ri.ucsal.br:8080/jspui/handle/prefix/2766.

Dias, M. J. L. E. Cchini, M. C. Cardoso, T. F.Orrico, S. R. P. \& Pereira, A. B. L. B. (2021). COVID-19 e nutrição. ULAKES Journal of Medicine. 1 (EE) 106117. http://revistas.unilago.edu.br/index.php/ulakes.

Dutra, A. de F. de F. de O. Dias, A. D. C. Araújo, D. G. de S. \& Silva, E. M. da. (2020). A importância da alimentação saudável e estado nutricional adequado frente a pandemia de COVID-19. Braz. J. of Develop. 6(9), 66464-66473. https://www.brazilianjournals. com/index.php/BRJD/article/view/16336/13361.

Ferraz, S. de O. (2021). Alimentação como auxiliar na recuperação pós-Covid-19. CPAH Science Journal of Health. 1(2), 40-46. https://cpahjournal.com.br/index.php/cpahofhealth/article/view/27/64.

Gois, B. P. Pereira, A. D. Lopes, K. L. S. \& Corgosinho, F. C. (2020). Suplementação e alimentação adequada no contexto atual da pandemia causada pela COVID-19. Revista Desafios. 7. COVID-12, https://sistemas.uft.edu.br/periodicos/index.php/desafios/article/view/8825/16733

Gomes, L. M. de F. (2020). A importância da alimentação saudável e estado nutricional adequado frente a pandemia de COVID-19. Braz. J. of Develop. Curitiba, 6(9), 66464-66473, https://www.brazilianjournals.com/index.php/BRJD/article/view/16336/13361.

Gomes Júnior, N. N. (2015). Segurança alimentar e nutricional e necessidades humanas. Editora Fundação Perseu Abramo.

Guimarães, L. M. \& Oliveira, D. S. (2014). Influência de uma alimentação saudável para longevidade e prevenção de doenças. Interciência Sociedade. 3(2). http://revista.francomontoro.com.br/intercienciaesociedade/article/view/68/56.

Lima Júnior, L. C. (2020). Alimentação saudável e exercícios físicos em meio à pandemia da COVID-19. Boletim de conjuntura (boca). 3(9), 33-41, 10.5281/zenodo.3988664. http://revista.ioles.com.br/boca/index.php/revista/article/view/62.

Lima, M. R. da S., \& Soares, A. C. N. (2020). Alimentação saudável em tempos de COVID-19: o que eu preciso saber? Braz. J. Hea. Ver. 3(3), 3980-3992 may./jun. 2020. https://www.brazilianjournals.com/index.php/BJHR/article/view/9537/8026.

Lima, R. de S. F. Neto, J. A. F. \& Farias, R. de C. P. (2015). Alimentação, comida e cultura: o exercício da comensalidade. Demetra: alimentação, nutrição saúde. Demetra, 10(3), 507-522. https://www.e-publicacoes.uerj.br/index.php/demetra/article/view/16072/13748.

Lima, L. W. Batista, M. C. C. Silvino, V. O. Moura, R. C. Mendes, I. L. Moura, M. S. B. Batista N. K. C. Silva, K. R. \& Barbosa, a. K. da. S. (2020). Importância nutricional das vitaminas e minerais na infecção da COVID-19. Research, Society and Development. 9(8), e804986103, 10.33448 / rsd-v9i8.6103. https://rsdjournal.org/index.php/rsd/article/view/6103.

Marconi, M.de A. \& Lakatos, E. M. (2018). Fundamentos de metodologia científica. Ed. Atlas. 
Martinelli, S. S. Cavalli, S. B. Fabri, R. K. Veiros, M. B. Reis, A. B. C. \& Santos, L. A. (2020). Estratégias para a promoção da alimentação saudável, adequada e sustentável no Brasil em tempos de Covid-19. Rev. Nutr. 2020,33:e200181.

Martins, M. do C. de C. Oliveira, A. S. da S. \& Sales, A. de C. C. (2020). Zinco e doenças respiratórias virais: efeito na infecção do novo coronavírus. JCS HUUFPI. https://periodicos.ufpi.br/index.php/rehu/article/view/794

Martins, M. C. C. \& Oliveira, A. S. S. (2020). Zinco, vitamina D e sistema imune: papel na infecção pelo novo coronavírus. Revista da FAESF. https://www.epublicacoes.uerj.br/index.php/demetra/article/view/16072/13748.

Nascimento, W. dos S. (2018). Segurança alimentar e nutricional e agricultura familiar: o caso de São Francisco do Conde, Bahia. Universidade da Integração Internacional da Lusofonia Afro-Brasileira (UNILAB). https://repositorio.unilab.edu.br/jspui/handle/123456789/1835.

Pires, R. R. (2020). A crise sanitária da COVID-19: propostas para o aperfeiçoamento da ação pública. Instituto de Pesquisa Econômica aplicada-IPEA. http://repositorio.ipea.gov.br/handle/11058/9839.

Reis, F. da S. \& Macagnan, F. T. (2020). Micronutrientes: uma revisão sobre a sua relação com o sistema imunológico, biodisponibilidade e fortificação dos alimentos. https://repositorio.ifsc.edu.br/handle/123456789/2290.

Rodrigues, H. de A. F. (2012). Alimentação como fonte de sociabilidade e de hospitalidade. In: SINAIS - Revista Eletrônica. Ciências Sociais. Vitória: CCHN, UFES, 12(1), 85 - 100. https://periodicos.ufes.br/sinais/article/view/5853.

Rodrigues, C. P. F. Fonseca, L. F. R. Morais, P. B. \& Neumann, K. R. S. (2021). O papel da vitamina D no sistema imunológico e suas implicações na imunidade inata e adquirida. Revista Interação. Interação, 21(1), 249-269. Recuperado de http://interacao.org/index.php/edicoes/article/view/150.

Santiago, M. B. \& Souza, M. L. R. de. (2020). Uma revisão sobre a deficiência de selênio e a suscetibilidade às infecções virais com ênfase particular no novo coronavírus. Braz. J. Hea. Ver. 3(5), 11509-11520. https://www.brazilianjournals.com/index.php/BJHR/article/view/16041/13135.

Sambuichi, R. H. R. Almeida, A. F. C. S. de. Perin, G. Spínola, P. A. C. \& Pella, A. F. C. O (2020). Programa de aquisição de alimentos (PAA): instrumento de dinamismo econômico, combate à pobreza e promoção da segurança alimentar e nutricional em tempos de COVID-19. IPEA. http://repositorio.ipea.gov.br/handle/11058/10032.

Silva, J. K. Prado, S. D, Seixas, C. M. (2017). A força do "hábito alimentar". Physis Revista de Saúde Coletiva. 27(4), 1065-1085, https://www.scielo.br/j/physis/a/7VGgLwJzNN3VVB8pwKLTNFS/abstract/?format=html\&lang=pt.

Silva, R. de C. R. Pereira, M. Campelo, T. Aragão, E. Guimarães, J. M. de M. Ferreira, A. JF. Barreto, M. L. \& Santos, S. M. C. dos. (2020). Implicações da pandemia COVID-19 para a segurança alimentar e nutricional no Brasil. Ciência Saúde Coletiva. 25(9):3421-3430, https://www.scielo.br/j/csc/a/mFBrPHcbPdQCPdsJYN4ncLy/?lang=pt\&format=html.

Silvino, V. O. Pereira, M. M. L. Moura, R. C. Batista, M. C. C. Rosa, B. V. Moura, E. H. de. Martins, M. C. C. \& Santos, M. A. P. (2019). Vitamina D e doenças infectocontagiosas na pandemia da COVID-19. Research, Society and Development, 9(7): 1-21, e771974614.

Vasconcelos, A. B. P. de A., \& Moura, L. B. A. de. (2018). Segurança alimentar e nutricional: uma análise da situação da descentralização de sua política pública nacional. Cad. Saúde Pública. 2018, 34(2): e 00206816. https://www.scielo.br/j/csp/a/zBLgfjqmvXSJKJhcLz8Gf4c/?lang=pt\&format=html.

Vaz, D. S. S. \& Bennemann, R. M. (2014). Comportamento alimentar e hábito alimentar uma revisão. Ed. Uningá Review.

Vidal, A. M. Dias, D. O. Martins, E. S. M. Oliveira, R. S. Nascimento, R. M. S., \& Correira, M. G. S. (2012). A ingestão de alimentos funcionais e sua contribuição para a diminuição da incidência de doenças. Cadernos de Graduação - Ciências Biológicas e da Saúde Aracaju. SERGIPE, 1(1), 43-52. https://periodicos.set.edu.br/cadernobiologicas/article/view/284. 